2.

\title{
Entgegnung auf das an mich gerichtete letzte Wort des Herrn Marchand (Heft 2 S. 37\%).
}

\author{
Von P. Grawitz in Greifswald.
}

Wenn ich nicht ausreichende Gründe bätte, die Autorität Marchand's auf dem Gebiete der Biologie mit Vorsicht und Misstrauen zu behandeln, so würde mich sein Urtheil, dass ich mit der Schlummerzellentheorie ein todtgebornes Kind vertheidigte, in aufrichtige Trauer versetzen. Ich vertheidige aber gar keine Schlummerzellentheorie; ich habe seit 4 Jahren immer mit derselben Entschiedenheit, wie in weinem Atlas der patbologischen Gewebelebre, stark betont, dass ich für die autochthone Bildung der sogenannten Wanderzellen aus Gewebsbestandtheilen eintrete; speciell für die Hornbaut habe ich vor 3 Jahren in den Dissertationen von Anders und Buddee die Ergebnisse der älteren Beobachter über das Hervorgehen der glänzenden protoplasmatischen Gebilde bestätigt und zu erweitern gesucht, und mich unter freier Loslösung vom Drucke herrschender Theorien immer wieder für incompetent zur Aufstellung einer eigenen Theorie erklärt. Alledem zum Trotz schreiben diejenigen meiner Fachgenossen, deren bittere Feindschaft ich mir durch die Bekämpfung der Immigrationstheorie zugezogen habe, consequent von meiner Schlummerzellentbeorie. Deswegen sollte es Herr Marchand nicht so übel nebmen, wenn wir gelegentlich von einer Befangenheit oder Voreingenommenheit unserer Gegner sprechen; es geschieht vielleicht ohne die Absicht der glücklichen Majorität, aber es geschieht, dass sich die vorsichtigen Unbetheiligten sagen: Schlummerzellentheorie ist eine moderne Verirrung, schweigen wir davon, wäbrend die Entstehung contractiler Zellen aus Gewebszellen oder deren Ausläufern ein durchaus discutables, - wenn nicht gar sympathisches Thema ist. Die Darstellung, dass ich neuerdings einen Rückzug antrete, wie M a r chand meint, mag wohl einem Wunsche seinerseits entsprungen sein, ibre Unrichtigkeit ist aber aus dem Schlussworte in meinem Atlas obne weiteres zu ersehen; seit Jahren vertheidigen wir das Hervorgeben von Wanderzellen aus dem Hornbautgewebe, wie es vor uns viele angesebene Beobachter gethan haben, die von dem modernen Medusenbaupte der Schlummerzellentheorie keine Ahnung hatten.

Wer die objectiv gehaltene Schilderung Rollet's über den Keratitisstreit 1872 in Stricker's Histologie liest, der findet, dass Rollet unter Anwendung von Joddämpfen in frischen Hornbäuten das Hervortreten der Wanderzellen aus dem Gewebe auf Grund eigener und fremder Beobachtungen als Thatsache beschreibt, während er das Eindringen fremder Wander 
zellen eben nur als eine Concession behandelt, deswegen gemacht, weil er die Möglichkeit einer Einwanderung nicht unbedingt widerlegen konnte. Wenn später die Thatsache durch die nur als möglich hingestellte Immigrations-Theorie (siehe Buddee's Arbeit) ganz in den Hintergrund gedrängt worden ist, so ist daran die ausserordentliche, durch keine ruhige Kritik im Zaume gehaltene Begeisterung Schuld, welche seiner Zeit der Co hn hei m' schen Theorie entgegengebracht worden ist. Man lese den Aufsatz von Billroth "Mancherlei über die morphologischen Veränderungen bei der Entzŭndung“, Wiener med. Jahrb., 1869, um sich zu überzeugen, wie einer der einflussreichsten, um die pathologiscbe Histologie verdientesten Forscher jener Zeit mit fliegenden Fahnen in das Lager der neuen Verkündigung übergegangen ist, wie er völlig davon überzeug $t$ ist, dass die kleinzellige Infiltration der Carcinome von Leukocyten geliefert wird, und wie diese Theorie für die Sarcome zwar schwer zu beweisen, aber doch sehr wahrscheinlich sei. Es hat meines Erachtens historischen Werth, wiederholt daran zu erinnern, dass Ende der 70 er Jahre oder später die Lehre weit verbreitet war, dass alle Regeneration von Leukocyten ausgebe, und dass $\mathrm{Cohnh} \theta \mathrm{im}$ und die auf seinen Pfaden wandelnden Pathologen zu einer ungeheuren Einschränkung dieser Theorie gelangten, als sie mit Eifer an die Untersuchung der concreten Objecte herantraten und aufhörten, Alles nach der Theorie a priori zu bestimmen. Auf diesem Wege hat Marchand Fortschritte gemacht, indem er auch bei der Einheilung von Fremdkörpern die Rolle der Leukocyten als eine untergeordnete erkannte, und es ist zu hoffen, dass auch die Keratitisfrage in diesen rückläufigen Strom einbezogen werden wird, wenn man - Verzeibung für das Wort - unbefangen, obne Theorie, an ihre Lösung herangehen wird, d. h. unter sorgfältiger Nachprüfung aller nicht allein pro, sondern auch contra bisher angeführten Beobachtungen, wie ich mir in jahrelanger Arbeit Mühe gegeben habe.

Marchand und sein Sebüler Goecke machen mir den Vorwurf, ich habe mit der Behauptung, eine Hasenhornhaut könne 12 Tage nach dem Tode des Thieres noch erbolungsfähig sein, obgleich oberflächlich Schimmelbildung entstanden war, eine $A$ uferweckung vom Tode verkündet. Ich sei so von einer Theorie eingenommen, dass ich die einfachsten Naturgesetze ausser Acht liesse. Der Vorwurf blinder Voreingenommenheit scheint also weniger verletzend $z u$ wirken, wenn er in schroffster Form gegen mich gerichtet wird, als wenn er sich in milder Form gegen die beati possidentes der vox populi richtet. Ohne meinen Empfindungen über diese Behandlungsweise Ausdruck zu geben, habe ich das rein sachliche Verfahren eingeschlagen, Notizen über das Fortleben einzelner Gewebsstücke nach dem Tode des ganzen Individuums zu sammeln ${ }^{1}$ ), Wenn Herr Marchand nicht verstanden hat, was für Beziehungen zur Biologie der Cornea die höchst lehrreiche historische Darlegung der Streitfrage über die Animalia resuscitantia hat,

1) s. Deutsche med, Wochenschr, 1897, No. 1 und 2. 
so will ich es hier wiederholen: 1) zeigt sie, dass Anguillulae, Rotatorien und Tardigraden, welehe nach den Ermittelungen neuerer Zoologen mitotische Kerntheilung ihrer Eier und im ausgewachsenen Körper Ganglien, Muskeln, Drüsenzellen enthalten, gleichwohl Temperaturscbwankungen von $95^{\circ} \mathrm{C}$. und längere Zeit Hitzegrade, bei denen Eiweiss coagulirt, ertragen können, obne abzusterben. Diese biologische Thatsache ist doch wohl beweisend dafür, dass man über Lebensfähigkeit einer Cornea nicht nach blossem Augenschein urtheilen darf. 2) lehrt die Geschichte dieser Thiere, dass im Verlaufe von 200 Jahren dieselbe Entdeckung etwa 5 Mal gemacht worden ist, und dass sie immer wieder bestritten und vergessen ist, weil sie mit dem populären Begriffe vom Leben und vom Tode nicht zu vereinigen war. Bis in die neueste Zeit hat man angenommen, dass die Behauptung, getrocknete Thiere könnten jahrelang leben und $78^{\circ}$, ja $100^{\circ}$ Hitze aushalten, allen Naturgesetzen widerstreite, und deshalb a priori absurd sei. Der gegen Needham 1743 geäusserte Spott, er bebaupte eine Auferweckung der Thiere vom Tode, hat die 1860 erfolgte endgültige Bestätigung seiner Beobachtungen nicht verbindert.

Von den anderen Beispielen, über welche ich und kürzlich Busse berichteten, führt Marchand das Ueberleben der Flimmerzellen an, welcbes Virchow an menschlichen Leichen 52 Stunden, P. M. Valentin an todten fauligen, fast zerfliessenden Schleimbäuten von Fröschen, Busse an fauligen Polypen bis zum 18. Tage beobachtete, wo doch gewiss stärkere Ptomaine wirkten, als bei der von Schimmel befallenen Hasenhornhaut.

Ausser den Cilienschwingungen sah $B$ usse noch andere Lebenserscheinungen, und forderte nunmehr Herrn Marchand auf, seinerseits die einfachen Naturgesetze nebst Begründung zu nennen, nach denen ein Fortleben 12 Tage lang a priori absurd sei. Diesem gewiss berechtigten Verlangen kommt Herr Marchand nicht einmal versuchsweise nach. Er lässt gesperrt drucken, dass ein Unterschied zwischen dem Fortleben der Flimmerzellen und der Erhaltung einer Proliferationsfähigkeit bestehe, was uns wirklich eben so wenig neu ist, als seine Bemerkung, dass die vitale Energie der einzelnen Gewebe sehr verschieden sei; davon kann sich Jeder aus meiner Rectoratsrede belebren, wo der verschiedenen Grade der Reaction und auch der einfachen regressiven Vorgänge als Beweis für die Erhaltung des Lebens Erwăhnung geschieht. Wir haben inzwischen einiges Neue über die Erbaltung der vollsten Proliferationsfäbigkeit ermittelt: Herr Dr. Grohé hat bereits beim Beginn seiner Nacbprüfung der Ollier'schen Versuche üppige Knorpel- und Markgewebsproliferation an Periost gefunden, das der Leiche eines 74 Stunden todten Kaninchens entnommen war. Die Angaben von L usk babe ich an einer Stelle mit dem Vermerke, „falls sie sich bewahrheiten sollten", angeführt; sie haben sich nicht bewabrheitet, - dagegen ist mir von einem Collegen, auf den meine biologischen Mittheilungen einen günstigeren Eindruck, als auf Herrn M archand, gemacht hatten, eine Reihe von unzw đideutigen Beweisstücken für mitotische Zellenvermehrung mensch- 
licher Gewebe vorgelegt worden, die 14 Tage und mebr abgetrennt gewesen waren.

An getrockneter Cornea habe ich nach 4 tägigem Lymphbade reichlicbe Mitosen in Hornbautkörperchen gefunden, so unglaublich der Befund a priori erscheinen mag; ich habe diese Objecte den Herren Waldejer und Bonnet gezeigt, bei ihnen kann sich Herr Marchand erkundigen, ob sie sich überzeugt baben, dass es gerade solche verzweigte Hornhautzellen waren, wie sie bei Regeneration daselbst vorkommen. Ich habe also nicht nur für Flimmerzellen, deren 18 tägiges Ueberieben ich übrigens keineswegs so gering schätze, wie Herr Marchand, und für die Erhaltung der Proliferationsfähigkeit ganz schlagende Beweise gebracht, ich habe die Uebereinstimmung der Bilder in der 12 Tage überlebenden Cornea mit denen der lebensfrisch transplantirten nachgewiesen, und muss es den Urtheile der Leser überlassen, ob die Behauptung von Marchand, ich hätte mit anderen Worten erklärt, dass Leichen lebendig seien, einen Ersatz für die schuldig gebliebene Mittheilung seiner einfachsten Naturgesetze bieten kann.

In meiner Nachprüfung der Versuche von Senftleben und Leber habe ich bewiesen, dass diese von allen Seiten mir als sicherste Stütze für Leukocyteneinwanderung gegen die Annahme einer autochthonen Bildung der Wanderzellen entgegengehaltenen Versuche von der ganz unbewiesenen Annabme ausgeben, dass die Hornbäute todt gewesen seien. Dass diese Annahme unbewiesen war, giebt Orth ohne Einschränkung zu; dass sie aber auch unrichtig war, geht aus dem oben bereits angeführten Vorkommen von Mitosen in solchen Hornhäuten bervor, die genau nach Leber's Angaben getrocknet und injicirt waren. Ich babe nun viele hunderte von Hornhautpräparaten seit Jahren gesammelt, welche zeigen, dass ganz frisch entnommene Froscbcornea im Lymphsacke gleiche Spiessformen annimmt, wie eine mehrere Tage überlebende Cornea sie zeigt. In einer gekochten oder auch nur eine Viertelstunde auf $60^{\circ}$ erwärmten Froschbornbaut babe ich nie solche Spiesse gefunden, voausgesetzt natürlich, dass sie nicht vorber schon darin zu seben waren. Ich babe daraus den Scbluss gezogen, dass in wirklich todtes Bornhautgewebe keine Zellen einwandern, von Froschcornea babe jch auch nie Bilder geseben, die mich zweifelbaft gemacht bätten. Dagegen geben gekocbte Hornbăute vom Scbwein oder Kaninchen - auch ohne Transplantation in ein Lympboad - ganz ausserordentlich mannichfaltige Vergoldungs- und Chromatinfiguren. Nach kurzem Kochen und Anheilung im Bauchfellsack habe ich unzweifelhafte Chromatinvermebrung, merkwürdige lange Spiralen, vollkommenen Zerfall des Gewebes in lauter chromatinähnlich tingirte Spiralstücke und Aebnliches beobachtet, und babe gesehen, wie von dem andringenden Granulationsgewebe diese zerfallenen Stellen durchwuchert und resorbirt werden. Es ist mir also nicht so überraschend, dass in einer Cornea, die angeblich todt ist, noch Veränderungen zu beobachten sind, die zwar total von den Spiessformen frisch transplantirter Hornhäute verschieden sind, die aber immerhin 
ganz gut gegen den Sat $z$,in todtes Hornhautgewebe wandert überhaupt unter keinen Umständen etwas ein “ angeführt werden können. Icb beab sichtige, über diese verschiedenen Formen in einiger Zeit eine möglichst objective Mittheilung zu machen, und verspreche, alsdann auf die Versuche von Saxer und Orth einzugehen; bis dahin bitte ich den geehrten Leser, sein Urtheil über die Beweiskraft der Widerlegung in suspenso lassen zu wollen. Meinen Streitpunkt mit Marchand bildet doch nicht die zugespitzte Frage, ob möglicherweise unter ganz besonderer Anordnung der Versuche Zellen in erweichte todte Cornea eindringen können, sondern die Frage, ob die Spiessfiguren, welche bei 12 Tage todten Hasen in Menge vergoldbar sind, eingewandert sind, oder ob sie rom Gewebe abstammen. Es nimmt mich Wunder, dass Marchand mit keinem Worte davon Notiz genommen hat, dass ich mich auch betreffs dieser Spiessfiguren auf sachkundige Fachleute berufen habe, dass es sich also doch verlohnt bätte, Objecte dieser Art herzustellen und anzuseben. Das principielle Vermeiden aller derjenigen Untersuchungsmethoden, durch welche man die Formveränderungen der Hornbautzellen ohne Leukocytennähe kennen lernt, kann auf die Dauer doch nicht aufrecht erhalten werden. Buddee hat sich in seiner ganzen Abhandlung, welche auf jahrelanger Arbeit beruht, auf den Standpunkt gestellt, den Marchand und $0 \mathrm{rtb}$ wiederhergestellt $z$ haben glauben, er nimmt die Möglicbkeit einer Immigration an, er führt trotzdem viele Thatsachen an, welche beweisen, dass die Spiessformen unter Bedingungen vorkommen, welche nach Cobnheim's ausdrücklicher Angabe die Einwanderung ausschliessen. Warum werden die Chromatinbildungen im Aetzschorf, von denen doch selbst Klemensciew icz zugiebt, dass es keine Lenkocyten sein können, nicht nachgeprüft? Von den riesenzellenähnlichen Protoplasmaballen hat Buddee gesagt, dass sio von älteren Patbologen, neverdings von Klemensciewic $z$, als eingewanderte confluirte Leukocyten angesprochen seien, während sie jetzt von den Schülern Marchand's für Abkömmlinge der Gewebszellen gehalten würden. Dies soll beweisen, wie unsicher alle diese Angaben selbst bei den Vertretern der Immigrationstheorie sind -; es mag sein, dass Marchand in dem Hervorheben dieser Thatsache eine an Naivetät grenzende Unbefangenbeit sieht. Ich glaube jetzt das Princip, nach dem die böcbst verschieden grossen, aber unter einander doch wieder äbnlichen Spiesse zu Stande kommen, begriffen zu baben, so dass ich das nächste Mal statt der Berufung auf Autoritäten positive Beweise für die autochthone Entstebung der Spiesso gegen die Deutung von aufmarschirten Leukocyten zu bringen hoffe. - -

Zur Erklärung dafür, dass ich geschrieben habe, Leber und Marchand fassten den Entzündungsprozess nicht, wie von Hippocrates bis Virchow üblich, als Reaction lebender Gewebe auf, sie erblickten in den Vorgängen der obne eigene active Proliferation einbeilenden Fremdkörper eine Analogie des Entzündungsprozesses, mit anderen Worten, sie behaupten die Möglichleit einer Entzündung obne Reaction lebender Gewebe, 
seien ibre Auslassungen wörtlich citirt (Leber S. 427): „Dass die Hornbaut bei diesen Vorgängen eine wesentlicb passive Rolle spielt, wird zur Evidenz erwiesen durch die von mir gemachte Beobachtung, dass dieselben Formen der eitrigen Infiltration, welche von der lebenden Hornhaut bekannt sind, nehmlich die Infiltration einer direct verletzten von der äusseren Oberfläche aus, und der Infiltrationsring durch Randeinwanderung anch an einer todten Hornhant im Innern des lebenden Thierkörpers auftreten. . . Wirkt ein Entzünduugsreiz in der todten Hornhaut in gleicher Weise als Attractionscentrum für die Leukocyten wie in der lebenden, so können die Elemente des Hornhautgewebes (bei der Keratitis nehmlicb) nicht in activer Weise betheiligt sein ". Kann man klarer den Gedanken einer Entzündung ohne Reaction lebender Gewebe aussprecben?

Büngner (Ziegler's Beitr. Bd. 19) hat, gleich wie Leber, den Fremdkörper nicht als "Reiz" in die Bauchböble gebracht, um Peritonitis zu studiren, sondern ebenso, wie Leber die Cornea mit Entzündungserregern injicirt (Terpenthin, Bakt. u. s. w.), um die Eiterinfiltration der todten Cornea selbst zu studiren, so hat auch Büngner Terpenthin, Bact. u. s. w. injicirt und in dem Eindringen verschiedener Zellenarten in das Centrum des bakterienhaltigen Schwammes die Analogie mit Entzündungsheerden erblickt. Was bei Leber Eiterinfiltration der Cornea ist, das ist hier Eiterinfiltration des Schwammes. Es heisst S. 55: „auch hier kann es keinem Zweifel unterliegen, dass sämmtliche Rundzellen, welche sich in diesem Stadium des Entzündungsprozesses im Fremdkörper ansammeln, ausnahmslos aus dem Blute stammende Leukocyten, mithin Exsudatzellen sind". S. 100: „Aus dieser Fäbigkeit der Exsudatzellen, Zerfallsstoffe und verschiedenartige Schädlichkeiten in sich aufzunehmen, und beseitigen zu können, indem sie, mit denselben beladen, aus dem Entzündungsgebiet (das ist der Fremdkörper!) wieder auswandern, erhellt die grosse Bedeutung der Phagocytose“. S. 122: "Gleich Marchand und unseren übrigen Yorarbeitern müssen auch wir bei der Betrachtung der Entzündungsvorgänge, welche sich an die Einheilung von Fremdkörpern anschliessen, zwei Phasen des Prozesses auseinanderhalten: - die Auswanderung farbloser Blutkörperchen aus den Gefässen und ihre weiteren Schicksale im Bereiche des Entzündungheerdes" (das ist wieder der Fremdkörper!) „und zweitens die Wucherungsvorgänge des Grundgewebes, welehe zur Bildung des Dauergewebes führen". Das ist also gewissermaassen schematisch die Lebre, dass die exsudative Entzündung entsteht durch Bakterien, welche Leukocyteneinwanderung bewirken; später setzen Regenerationswucherungen in der Nachbarschaft ein, welche zur Narbenbildung führen, und alles vollziebt sich in einem Schwamm. Wenn diese Versuche nicht die passive Rolle des Entzündungsbeerdes gegenüber der activen Rolle eingewanderter Zellen beweisen sollen, so liegt die Schuld für ein Missverständniss nicht auf meiner Seite. 
Nicht um die Schlummerzellentheorie handelt es sich. Die Auffassung, dass Einwanderungs- und Organisationsvorgänge in einem implantirten Schwammstückchen gleichzusetzen sind der entzündlichen Reaction lebenden Gewebes, das ist das, was ich als die Grenzscheide zwischen Virch ow'scher und Cohnheim'scher Schule bezeichne. Wenn erst das Hervorgehen massenhafter Wanderzellen aus dem Corneagewebe wieder als Thatsache anerkannt sein wird, wie es 1872 war, dann wollen wir weiter darüber streiten, aus welchen Bestandtheilen des Gewebes sie hervorgehen.

3.

Eine historische Erinnerung an vergangene ärztliche Zeiten.

Von Dr. Laudon in Elbing.

In der Elbingisch-Preussischen Chronick Christoph Falk's (herausgegeben von Toeppen, Leipzig, Dunker \& Humblot, 1879) findet sich als besonderer Abschnitt die kleine, dem Rathe der Stadt überreichte Schrift: „Der Stat Elbing lobspruch sampt irer umligenden Landschaft beschreibung, $1548^{\text {", }}$, die wobl nur ein locales Interesse hat. Doch liest man darin einige Verse ${ }^{1}$ ), die auf ärztliche Verbältnisse einiges Licht werfen. Wenn auch das marktschreierische Treiben der Aerzte jener Zeit geschichtlich festgestellt ist, so mag die Thatsache, dass es hier im Osten sich eben so breit gemacht babe, wie im Deutschen Reich, erwäbnenswerth sein.

Falk, der die Provinz Preussen, eine ibm unbelannte Gegend, durchreist, bat als Reisementor den Gott Mercur zur Seite, der, weil allwissend, auf jede an ihn gestellte Frage, sofort eine aufklärende Antwort zu geben bereit und im Stande ist.

Die Verse lauten:

Ich sprach, was ist das vor ein man,

Der vor dem schoenen haus (Rathhaus) thut stan

Und also hatt ein gros geschrey

Mit seiner kleinen Kramerei?

Er hat ja viel vor sich der zen,

Las uns hin zu im neher gehn.

Der gottes bot sagt mir zur hand

Es ist ein artzt aus fernen land.

1) Seite 200 . 